\title{
Unterschenkelödeme
}

\section{Beidseitige oder einseitige Schwellung?}

VoN M. GULICH

Eine 66-jährige Rentnerin klagt anlässlich eines Routinebesuchs in der Praxis über seit Monaten zunehmendes „Wasser in den Beinen", das abends schlimmer würde. Zusätzlich berichtet sie über Atemnot bei Belastungen.

\section{Woran sollte man denken?}

- Unterschenkelödeme sind ein häufiges Symptom in der Primärversorgung. Da auch vollkommen gesunde Personen nach längerem Sitzen (Flugreise), langem Stehen (Verkaufspersonal) oder nach Immobilisierung

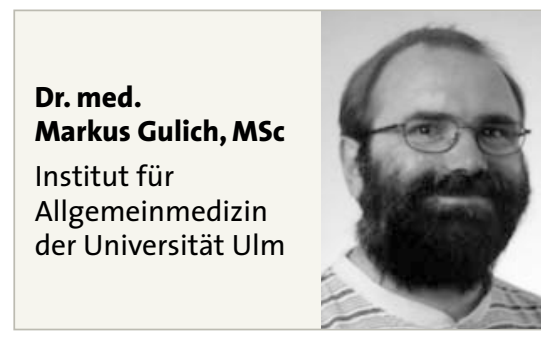

eine deutliche Ödemneigung entwickeln können, ist die Abgrenzung zu Unterschenkelschwellungen mit pathophysiologischer Relevanz (v.a. bei älteren Patienten) gelegentlich schwierig. Hier hilft eine sorgfältige Anamnese weiter.
- Die überwältigende Mehrzahl der Patienten mit (meist symmetrischen) Unterschenkelödemen weist als Ursache entweder eine sich entwickelnde Herzinsuffizienz oder eine chronisch venöse Insuffizienz auf.

- Gefürchteter, abwendbar gefährlicher Verlauf beim einseitigen bzw. asymmetrischen Ödem ist die tiefe Beinvenenthrombose (die meist mit spontanen oder Belastungsschmerzen einhergeht). Ein einseitiges Unterschenkelödem mit Entzündungszeichen (Rötung, Erwärmung, Labor) signalisiert meist ein Erysipel.

- Kalziumantagonisten vom Nifedipintyp können symmetrische Beinödeme auslösen. 
- Deutlich ausgeprägte beidseitige Unterschenkelödeme sind das klinische Leitsymptom des nephrotischen Syndroms. Die Ödembildung ist Folge einer Proteinurie (> 3,5 g/d) und der daraus resultierenden Hypalbuminämie.

- Seltene Ursachen sind mechanische Abflussbehinderung in den Beckenvenen oder der Vena cava (z. B. durch Tumoren), ein symmetrisches präti-

biales Myxödem im Rahmen einer Schilddrüsenerkrankung oder eine sympathische Reflexdystrophie als Hüfte-Fuß-Syndrom nach akutem zerebralem Insult.

\section{Was kann/sollte man tun?}

- Wesentlichstes Ziel der ersten hausärztlichen Beurteilung von Unterschenkelödemen ist der hinreichend sichere Ausschluss eines abwendbar

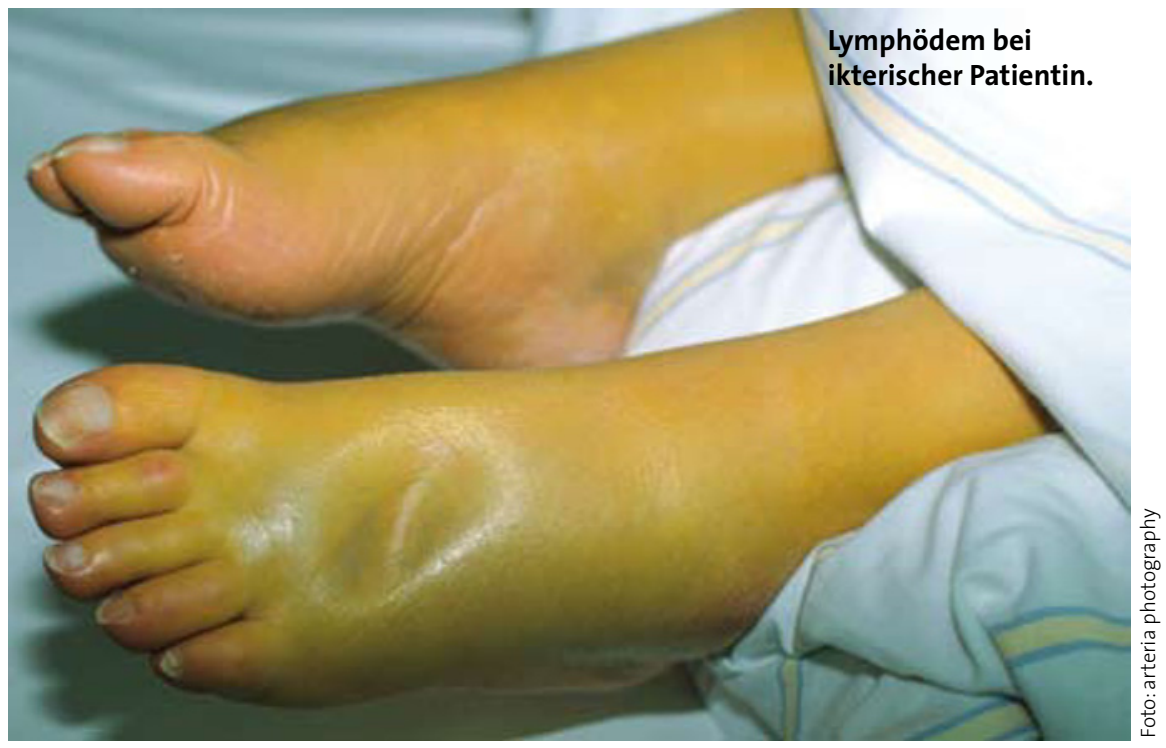

Tabelle 1

\section{Anamnese}

Anamnesefrage

Akuter Beginn?

Tagesrhythmik?

Symmetrie?

\section{Klärung folgender Fragen}

Beinvenenthrombose akut: Schmerzen spontan oder bei Belastung

Ödeme bei Herzinsuffizienz

Symmetrisch: z.B. Herzinsuffizienz, pulmonale Hypertonie, Nierenerkrankung (nephrotisches Syndrom), Leberzirrhose, Hypoalbuminämie, Mikroangiopathie, z. B. bei Diabetes, untere Einflussstauung bei Vena-cavaVerschluss

Asymmetrisch: Thrombose, chronisch venöse Insuffizienz, Lymphabflussstauung, mechanisches Abflusshindernis

Medikamente/UAW? Kalziumantagonisten (Nifedipin-Typ), ACE-Hemmer, Glitazone, Mineral- oder Glukokortikoid, lang anhaltender Laxanziengebrauch, Absetzen von Diuretika

Akutereignis? Sturz/Trauma: Fraktur/Kontusion, posttraumatisches Ödem Zerebraler Insult: sympathische Reflexdystrophie (selten)

Begleitsymptome? Belastungsdyspnoe, Tachypnoe, Tachykardie: Herzinsuff. Subkutane Sklerosierung: chronisch venöse Insuffizienz Entzündungszeichen: Erysipel gefährlichen Verlaufs (Thrombose) und eine erste ätiologische Zuordnung.

- Die körperliche Untersuchung kann systemische (symmetrisch) von lokalen (einseitig bzw. deutlich asymmetrisch) Ödemen unterscheiden, ggf. durch Umfangsmessung.

- Ein leicht zu erhebendes klinisches Zeichen einer tiefen Beinvenenthrombose ist z. B. das Homan-Zeichen (Wadenschmerz bei Dorsalflexion des Fußes, Sensitivität $<50 \%$, Spezifität 50-90\%). Es darf jedoch nicht übersehen werden, dass klinische Zeichen keine ausreichende Spezifität für einen Thromboseausschluss haben. Bei entsprechend ausgeprägtem Verdacht ist eine Ultraschall-Doppler-Untersuchung indiziert.

- Liegt ein klinischer Verdacht auf eine Herzinsuffizienz vor, ist ein abgestuftes diagnostisches Vorgehen entsprechend der hausärztlichen Leitlinie Nr. 9 „Herzinsuffizienz" der DEGAM sehr zu empfehlen.

- Im Vordergrund der therapeutischen Bemühungen steht selbstredend die adäquate Therapie der Grunderkrankung. Akut lebensbedrohliche Situationen (z. B. akute Herzinsuffizienz) erfordern die unverzügliche stationäre Einweisung. Die Mehrheit der Erkrankungen, die Unterschenkelödeme verursachen, kann jedoch ambulant abgeklärt und behandelt werden. Für spezifische Untersuchungen ist oft die Kooperation mit entsprechenden Spezialisten angezeigt.

\section{Literatur beim Verfasser}

\section{Anschrift des Verfassers:}

Dr. med. Markus Gulich, MSc

Institut f. Allgemeinmedizin der Universität Helmholtzstraße 20

D-89069 Ulm

Tel.: 0731/500-31103, Fax: 0731/500-31109

E-Mail:markus.gulich@uni-ulm.de

\section{Keywords}

Lower limb oedema - Breathlessness - Congestive heart failure - Kidney failure-Venous insufficiency 